\title{
Experiments on rebounding slow impacts under asteroid conditions
}

\author{
Kolja Joeris ${ }^{1, *}$, Laurent Schönau ${ }^{1}$, Lars Schmidt ${ }^{1}$, Matthias Keulen ${ }^{1}$, Vrinda Desai ${ }^{2}$, Philip Born ${ }^{3}$, and Jonathan E. \\ Kollmer $^{1}$ \\ ${ }^{1}$ Experimentelle Astrophysik, Universität Duisburg-Essen, Lotharstr. 1-21, 47057 Duisburg, Germany \\ ${ }^{2}$ Dept. of Physics, 2401 Stinson Drive, North Carolina State University, Raleigh, NC 27695, USA \\ ${ }^{3}$ DLR, Institut für Materialpyhsik im Weltraum, Linder Höhe, 51147 Cologne, Germany
}

\begin{abstract}
We present a newly developed experiment for the examination of low-speed impacts under asteroid conditions. More specifically, our experimental setup enables us to simulate a very clean milligravity environment under vacuum, in which projectiles are shot at a granular bed at several $\mathrm{cm} / \mathrm{s}$. This granular bed consists of irregularly formed basalt particles with different size distributions. The experiment is carried out in the Bremen drop tower in its catapult mode, granting more than $9 \mathrm{~s}$ microgravity. Here, we discuss the setup and assess its performance.
\end{abstract}

\section{Introduction}

The surfaces of rubble-pile asteroids are covered in regolith of a variety of sizes. In some cases like for the asteroid Itokawa, the size distribution of regolith is not uniform across the surface [1]. More precisely, some areas are dominated by finer grains, while other areas are covered mainly by larger rocks. A comprehensive explanation for this sorting of grain sizes has yet to be found, but a number of hypotheses have been developed [2-4]. Among the mechanisms discussed is the so called brazil-nut-effect (BNE). Matsumura [3] suggests, asteroids might behave similarly to a box of cereals in which, when shaken, the largest constituents float to the top. Another competing explanation is what is called the ballistic-sorting-effect (BSE) [2]. In this approach, the sorting is assumed to happen not in the bulk material but on the asteroid surface where impacting particles sort themselves through different rebound behavior depending on the initial surface topology.

While there are a number of experiments on ejecta generation by granular impacts in the context of asteroids and planetesimals $[5,6]$ or on the gravity dependence of the BNE [7], little experimentation has been performed on the ballistic sorting hypothesis under realistic conditions. Here we detail an experiment that is able to mimic the granular surface of a rubble pile asteroid. We design our experiment such that it can examine the impact kinetics of slow projectiles into a regolith surface under conditions realistic for asteroid surfaces. Measuring if and how a slow impactor rebounds from an asteroid surface can be used to set practical limits on the role ballistic sorting plays in shaping an asteroids surface [8]. Creating realistic asteroid conditions is an engineering challenge since, besides a strong vacuum and the use of realistic materials, one needs

*e-mail: kolja.joeris@uni-duisburg-essen.de

A video is available at https://doi.org/10.48448/zzjt-j675 to provide a constant undisturbed low level of gravity on the order of $10^{-3}$ to $10^{-4} \mathrm{~g}$ without the use of a centrifuge that would imprint unwanted Coriolis forces on particles traversing the test volume.

\section{Experimental setup}

The idea of our experimental setup is as follows: we use the ZARM Bremen drop tower [9] to generate an environment with low residual gravity $\left(<10^{-6} \mathrm{~g}\right)$ where we mount a vacuum chamber onto a finely controlled linear stage which can provide a small constant acceleration. Inside the vacuum chamber, there is a launcher mechanism and a granular bed onto which test particles can be accelerated. At the drop tower the setup is placed into a capsule which is catapulted upwards inside a 120 meter long evacuated pipe and then falls down again, yielding a total of 9.3 seconds of free fall time and thereby microgravity inside the capsule.

\subsection{Vacuum chamber}

The aluminium vacuum chamber, shown in Fig. 1 holds a sample container of $10 \mathrm{~cm}$ diameter and a variable height between 2 to $5 \mathrm{~cm}$ in which crushed basaltic rock of various size distributions are placed to mimic the asteroid surface. This container is covered by a hatch, which prevents the regolith from escaping the container before the start of the experiment. The front and back sides of the chamber feature large glass windows, enabling a full view into the observation volume. Additional windows are located on the top of the chamber. Here, two LEDs are mounted for illumination. Attached to the top of the chamber is the impactor launcher. This launcher consists of a set of loaded springs that each accelerate a brass piston which in turn 


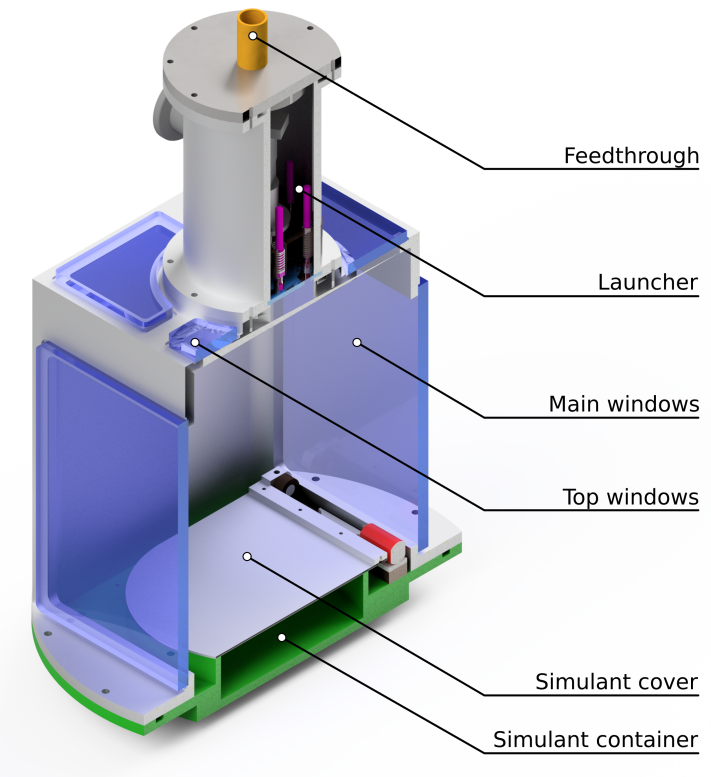

Figure 1. Schematic image of the experiment vacuum chamber. After the cover is opened an impactor is hauled onto the simulant by the launcher, producing ejecta which then can expand into the chamber.

pushes the sample impactor. The launch speed can be adjusted using screws set to pre-load the spring by a desired amount. Initially the impactor is held in place by a mechanical shutter. Once the shutter opens the test particle is launched onto the granular bed. This launcher has the capacity to hold six impactors with a diameter of up to $5 \mathrm{~mm}$ each. The overall system has a modular design, so the launcher as well as the vacuum chamber can easily be exchanged. This allows for different samples and granular beds to be tested. We also developed a version of the launcher that replaces the springs with electronically controlled solenoids for better repeatability This more precise launcher however can only hold one test particle. The necessary quality of the vacuum is determined by the condition that any residual air drag might not significantly influence particle motion. In this experiment we achieve an ambient pressure of less than 0.1 mbar, which is sufficient for the particle sizes used.

\subsection{Camera Systems}

Experiment data is captured by two camera systems. The main camera system is a Photron Fastcam MC-2 ${ }^{T M}$ with a resolution of $512 \times 512 \mathrm{px}$ and set to record between 125 and $250 \mathrm{fps}$ depending on the details of the experiment. The camera is positioned to look through the main side wall window, parallel to the granular surface. An exemplary sequence of frames from the main camera is shown in Fig. 2. To capture the 3-dimensional motion of the impactor, its rebound and any ejecta generated, a pair of stereo cameras is mounted on top of the vacuum chamber $(\mathrm{Pi}-\mathrm{Cam} \mathrm{V} 2$ with a resolution of $1920 \times 1080$ px at 25

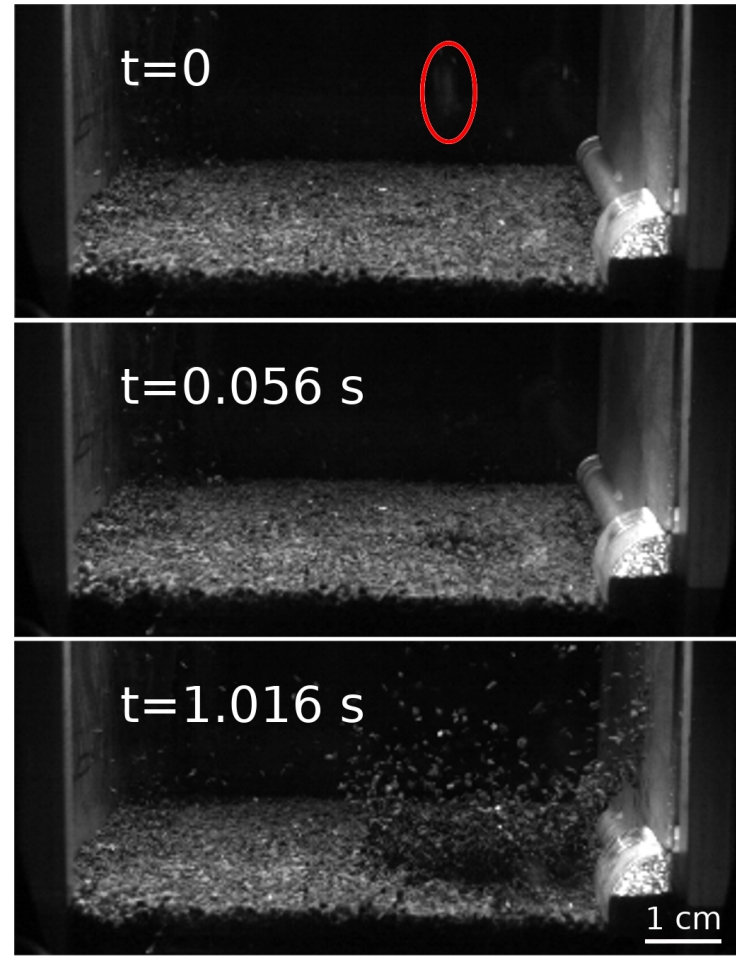

Figure 2. Image sequence captured by the main camera. Top: impactor, an approx. $3 \mathrm{~mm}$ irregularly shaped basalt bead (highlighted by red ellipse) moving towards the granular bed consisting of 0.5 to $1 \mathrm{~mm}$ sized crushed basalt. Note that the impactor appears blurred due to its speed of $600 \mathrm{~mm} / \mathrm{s}$. Middle: Moments after the impactor hits the surface. Bottom: One second after the impactor hits the granular surface an ejecta plume is clearly visible.

fps for the first $1 / 3$ of experiments and Basler a2A259060ucBAS with $1200 \times 1200 \mathrm{px}$ at $45 \mathrm{fps}$ for the other $2 / 3$ ).

\subsection{Simulant and Impactors}

For our simulated asteroid surface we use three types of material. 1) Crushed basalt with particle sizes ranging 0.5 to $1 \mathrm{~mm}$. 2) Crushed basalt with particle sizes ranging 1 to $3 \mathrm{~mm}$. 3) Finely powdered JSC MARS-1 simulant. The impactors are irregularly shaped pieces of basalt with a mass of approximately 40 to $50 \mathrm{mg}$ and a maximum edge length of $3.2 \mathrm{~mm}$.

\subsection{Gravity Generator}

In order to generate a gravity level similar to those found on the surface of asteroids, where the escape velocities can be as low as a few cm/s [10,11], the vacuum chamber is placed into an accelerated frame of reference. This is achieved by using a computer controlled high precision linear translation stage (Thorlabs DDS 220/M) with a 3phase direct drive onto which the chamber is mounted. Here the small gravitational acceleration on the asteroid is of advantage: taking into account that the maximum duration of the experiment in the drop tower is less than 10 seconds and assuming an acceleration smaller than $10^{-2} \frac{\mathrm{m}}{\mathrm{s}^{2}}$ 
$\left(10^{-3} \mathrm{~g}\right)$, one only needs a maximum travel distance of less than $0.5 \mathrm{~m}$ (which is the maximum travel space available in the drop capsule). When dealing with such small accelerations great care needs to be taken to avoid introducing any kind of distortion. Here, we take several measures to minimize any such distortion: 1 . Only the vacuum chamber with the launching mechanism is placed onto the translation stage, so the stage can be operated well below its load limit, leading to more load reserves for active control. 2. Reducing the amount of wiring and hosing connected to the vacuum chamber while it is translated. Since we need a high vacuum inside the chamber, it must be connected to a constantly running pump leading up to the experimental run. To not disturb the motion during the experiment itself, the vacuum line is automatically disconnected and the chamber sealed with a magnetic valve. Also, to avoid any mechanical interference by the camera wiring, we attach the main camera to a second translation stage, running synchronously and parallel to the stage with the experiment chamber.

\subsection{Landing}

The drop tower capsule lands in a bed of Styrofoam beads where it is strongly decelerated. Early tests indicated that this puts a lot of strain onto the translation stage holding the vacuum chamber, which in turn created a need for extensive maintenance after each experimental run. In order to preserve the integrity of our experimental assembly and reduce maintenance we added a pneumatic brake to the translation stage with the vacuum chamber. This small upgrade proved to be very effective and increased the translation mechanisms reliability to $100 \%$ over more than 10 repetitions of the experiment.

\subsection{Experiment procedure}

Several hours before the drop capsule is launched, the vacuum pump is switched on to evacuate the sample container and remove any residual out-gassing of the granular bed. Shortly before the catapult launch, the vacuum pump is stopped, the magnetic valve is closed, and the evacuation-line connector is withdrawn from the chamber. The pneumatic brake is fastened at this point, firmly locking the position of the gravity generating stage. The catapult launches the drop tower capsule and the acceleration from this launch introduces oscillations in the experimental setup, so the brake is held active for another $1.2 \mathrm{~s}$. Once the brake is released, the motion of the translation stages and camera systems are triggered. We initially start with an acceleration of $10 \frac{\mathrm{mm}}{\mathrm{s}^{2}}\left(10^{-3} \mathrm{~g}\right)$ to let any parts of the granular bed which might have become fluidized from the launch oscillations settle. After $1 \mathrm{~s}$ the acceleration is reduced to match the targeted asteroid-gravity. Now the sample container's cover is opened and the impactor is released and launched towards the granular bed. After the impact, to secure the experiment chamber for the landing deceleration of the drop tower capsule, the translation stage is returned to its starting position and firmly secured by the pneumatic brake again.

\section{Performance}

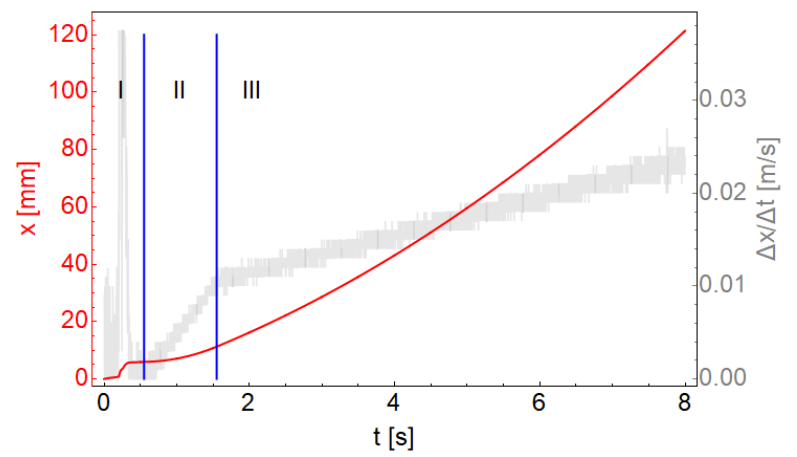

Figure 3. Red: Position of the experiment chamber relative to the drop capsule as measured by the Hall-effect position encoder. Gray: Position differences between two adjacent time steps in the red data set, indicating the instant velocity. Three regimes of experiment operation can be seen: I) Perturbation caused by the drop tower catapult launch. II) Initial acceleration phase (10.0 $\mathrm{mm} / \mathrm{s}^{2}, 10^{-3} \mathrm{~g}$ ) to consolidate the sample surface. III) Simulated asteroid gravity of $2.0 \mathrm{~mm} / \mathrm{s}^{2}\left(2 \times 10^{-4} \mathrm{~g}\right)$

Mimicking realistic asteroid conditions relies on achieving a stable low gravity environment, ideally without any g-jitter as would occur on parabolic flights or the space station [12]. Here the quality of our gravity level is determined by how precisely we can control the movement of the gravity generating stage. To evaluate this we attach a Hall-effect based position encoder to the stage so we can capture its position with $1 \mu \mathrm{m}$ accuracy at a sampling rate of $1 \mathrm{kHz}$. Fig. 3 shows the position of the vacuum chamber relative to the drop capsule exemplary for one experiment, along with the instant velocity of the stage. Three time intervals can be distinguished. In the first interval, the position shifts abruptly and the velocity peaks. This illustrates the initial oscillation caused by the drop tower catapult launch. Activating the pneumatic brake eliminates this initial peak.

In the second phase, we first drive the system at high ac-

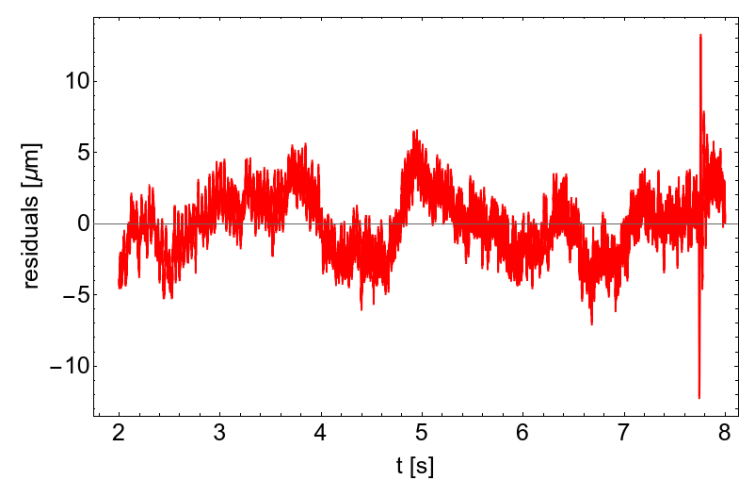

Figure 4. Differences between expected and measured position of the gravity generating stage as shown in Fig. 3, phase III. The deviation from an ideal parabola typically stay below $5 \mu \mathrm{m}$.

celeration to prepare the granular bed. In the third phase 
we settle on the gravity level desired for the specific experiment.

To evaluate the linear stage's performance, we fit a ballistic arc to the position data in phase III. Fig. 4 shows the residuals of this fit, that is, the deviation of the stage motion from the free fall motion at the set gravity level. These deviations typically lie well below $5 \mu \mathrm{m}$, with only one peak exceeding $10 \mu \mathrm{m}$. This proofs that the stage can position itself with high precision under the conditions inside the droptower capsule. This highly precise positioning in turn indicates the stage's ability to precisely follow a set acceleration and provide a stable low gravity environment. To evaluate the performance of the impactor launch system we use image data from all cameras to track the impactor in 3 dimensions. To study slow impacts the launcher should be able to generate a range of velocities. For our experiment we were able to achieve impact velocities in an interval from $30.71 \mathrm{~mm} / \mathrm{s}$ to more than $600 \mathrm{~mm} / \mathrm{s}$.

\section{Results}

We track the impactor and determine its velocity in 3 dimensions before and after the impact. For this tracking we use image data from the frontal camera system (see Fig. 2 ) as well as data from the top cameras. From these velocities, we can then compute a coefficient of restitution (COR). We average the COR for impacts of different velocities on each of the tested granular beds. We find that for the granular bed made from particles of similar size as the impactor ( 1 to $3 \mathrm{~mm}$ irregular shaped crushed basalt) we get a mean COR of 0.07 , for the granular bed of 0.5 to $1 \mathrm{~mm}$ sized particles we get a mean COR of 0.15 and for the finely powdered granular bed a COR of 0.80 . These results show a clear trend of higher coefficients of restitution, that is, a more elastic rebound, for impacts into finer material.

\section{Discussion}

We successfully developed an experimental setup to mimic the conditions on the surface of a rubble pile asteroid. Our experiments have confirmed that a computer controlled linear stage can generate stable, undisturbed, asteroid level gravitational accelerations inside a drop tower capsule. In a first test of the apparatus we find that the coefficient of restitution of slowly impacting particles is a function of grain size distribution of the granular bed. We can speculate that with the lack of gravitational confining pressure the smaller particles experience larger interparticle van-der-Waals forces $[10,13,14]$, thereby becoming a harder, more elastic target for the impactor. More experiments are necessary to map out this grain size dependent COR since one would expect it to also become high for solid targets much larger than the impactor. As discussed in the introduction, a better understanding of these impact kinetics is necessary to asses the applicability of various proposed models for explaining observed surface features on asteroids. Besides addressing these basic questions of asteroid formation, this kind of experimental setup might also be helpful in testing tools to properly handle granular materials on the surface of a rubble pile asteroid $[15,16]$.

We acknowledge helpful discussions with Tobias Steinpilz, Jens Teiser and Gerhard Wurm as well as technical support by Manfred Aderholz. This work was supported by the DLR Space Administration with funds provided by the Federal Ministry for Economic Affairs and Energy (BMWi) based on a decision of the German Federal Parliament under grant number 50WM1943.

\section{References}

[1] A. Fujiwara, J. Kawaguchi, D.K. Yeomans, M. Abe, T. Mukai, T. Okada, J. Saito, H. Yano, M. Yoshikawa, D.J. Scheeres et al., Science 312, 1330 (2006)

[2] T. Shinbrot, T. Sabuwala, T. Siu, M.V. Lazo, P. Chakraborty, Phys. Rev. Lett. 118, 111101 (2017)

[3] S. Matsumura, D.C. Richardson, P. Michel, S.R. Schwartz, R.L. Ballouz, Mon. Not. the R. Astron. Soc. 443, 3368 (2014)

[4] A.J. Dombard, O.S. Barnouin, L.M. Prockter, P.C. Thomas, Icarus 210, 713 (2010)

[5] J. Brisset, J. Colwell, A. Dove, S. Abukhalil, C. Cox, N. Mohammed, Prog. Earth Planet. Sci. 5, 1 (2018)

[6] J. Brisset, C. Cox, S. Anderson, J. Hatchitt, A. Madison, M. Mendonca, A. Partida, D. Remie, Astron. Astrophys. 642, 13 (2020)

[7] C. Güttler, I. von Borstel, R. Schräpler, J. Blum, Phys. Rev. E 87, 044201 (2013)

[8] T. Bogdan, J.E. Kollmer, J. Teiser, M. Kruss, G. Wurm, Icarus 341, 113646 (2020)

[9] ZARM Drop Tower Bremen User Manual (2012)

[10] P. Sánchez, D.J. Scheeres, Meteorit. Planet. Sci. 49, 788 (2014)

[11] K.E. Daniels, in Asteroids: Prospective Energy and Material Resources, edited by V. Badescu (Springer, Berlin, Heidelberg, 2013), pp. 271-286

[12] T. Steinpilz, G. Musiolik, M. Kruss, F. Jungmann, T. Demirci, M. Aderholz, J.E. Kollmer, J. Teiser, T. Bila, E. Guay et al., Rev. Sci. Instrum. 90, 104503 (2019)

[13] P. Sánchez, D.J. Scheeres, Icarus 338, 113443 (2020)

[14] D.J. Scheeres, C.M. Hartzell, P. Sánchez, M. Swift, Icarus 210, 968 (2010)

[15] J.E. Kollmer, S. Lindauer, K.E. Daniels, Digging on Asteroids: a Laboratory Model of Granular Dynamics in Microgravity, in ASCE Earth and Space 2016 Conference (Orlando, FL, 2016)

[16] J. Featherstone, R. Bullard, T. Emm, A. Jackson, R. Reid, S. Shefferman, A. Dove, J. Colwell, J.E. Kollmer, K.E. Daniels, Probe-insertion exhibits gravity-dependent stick-slip dynamics in experiments on a model system for regolith surfaces (2020), arXiv:2011.12890 